\title{
A gene for ataxic cerebral palsy maps to chromosome 9p12-q12
}

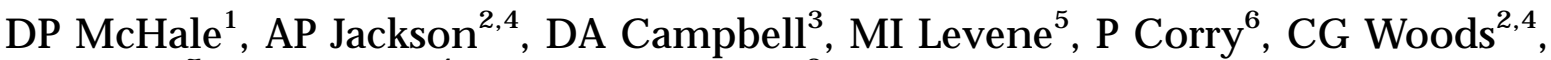 \\ NJ Lench ${ }^{7}$, RF Mueller ${ }^{4}$ and AF Markham ${ }^{2}$
}

\begin{abstract}
${ }^{1}$ Pharmacogenetics Unit, Central Research, Pfizer Ltd, Sandwich, Kent; ${ }^{2}$ M olecular Medicine Unit, University of Leeds, St James's University Hospital, Leeds; ${ }^{3}$ SmithKline Beecham Pharmaceuticals, Harlow, Essex; ${ }^{4}$ Yorkshire Regional Genetics Service, St James's University Hospital; ${ }^{5}$ Department of Paediatrics, Leeds General Infirmary, Leeds; ${ }^{6}$ Child Development Centre, St Luke's Hospital, Bradford; ${ }^{7}$ Oxagen Ltd, Abingdon, Oxfordshire, UK
\end{abstract}

\begin{abstract}
Cerebral palsy (CP) has an incidence of approximately 1 in 750 births, although this varies between ethnic groups. Genetic forms of the disease account for about $2 \%$ of cases in most countries, but contribute a larger proportion in certain sub-types of the condition and in populations with a large proportion of consanguineous marriages. Ataxic cerebral palsy accounts for 5-10\% of all forms of CP and it is estimated that approximately $50 \%$ of ataxic cerebral palsy is inherited as an autosomal recessive trait. We have identified a complex consanguineous Asian pedigree with four children in two sibships affected with ataxic cerebral palsy and have used homozygosity mapping to map the disorder in this family. A genome-wide search was performed using 343 fluorescently labelled polymorphic markers and linkage to chromosome 9p12-q12 was demonstrated. A maximum Lod score of 3.4 was observed between the markers D9S50 and D9S167 using multipoint analysis, a region of approximately 23cM. We have identified a family that segregates both ataxic CP and ataxic diplegia and have mapped the genetic locus responsible in this family to chromosome 9p12-q12. The identification of gene(s) involved in the aetiology of CP will offer the possibility of prenatal/premarital testing to some families with children affected with the disorder and will greatly increase our understanding of the development of the control of motor function. European Journal of Human Genetics (2000) 8, 267-272.
\end{abstract}

Keywords: cerebral palsy; aetiology; genetics; homozygosity mapping

\section{Introduction}

Cerebral palsy (CP) is defined as a non-progressive but not unchanging disorder of posture or movement caused by an abnormality of the brain, first evident from the stage of rapid brain development. ${ }^{1}$ Although CP can be classified according to the specific type of movement disorder observed and the distribution of limbs involved, ${ }^{2}$ it is a descriptive term for a neurological symptom complex and is aetiologically very heterogeneous. The phenotype seen in each individual patient reflects the combined effects of the type of insult as

Correspondence: Dr Duncan P McHale, Pharmacogenetics Unit, Central Research, Pfizer Ltd, Ramsgate Rd, Sandwich, Kent, CT13 9NJ, UK. Tel: + 44 1304 648921; Fax: +44 1304655614;

E-mail: Duncan-Mchale@sandwich.pfizer.com

Received 23 April 1999; revised 6 October 1999; accepted 26 October 1999 well as the timing of that event. The periventricular watershed areas of the brain are particularly vulnerable to disruption between 26 and 36 weeks' gestation leading to periventricular leucomalacia in babies born before 36 weeks' gestation. In contrast to this, the basal ganglia, hypothalamus, and brainstem are prone to damage during prolonged periods of hypoxia/anoxia during delivery. ${ }^{3}$ Genetic forms of $\mathrm{CP}$ account for approximately $2 \%$ of all cases of CP but this proportion varies markedly between different sub-types and between sample populations depending upon the degree of consanguinity within the population being studied. ${ }^{4,5}$

Ataxic CP accounts for approximately $5-10 \%$ of all cases of $\mathrm{CP}^{6}{ }^{6}$ It can be further sub-divided into ataxic diplegia and simple ataxia according to the presence or absence of spasticity in the lower limbs. ${ }^{7}$ It is estimated that up to $50 \%$ of cases with ataxic CP are due to a single gene disorder. ${ }^{1}$ The 
majority of cases are inherited as an autosomal recessive disorder but families with autosomal dominant and X-linked inheritance patterns have been described. ${ }^{4,7,8}$ Several syndromic forms of congenital ataxia are well documented and include the Joubert, Marinesco-Sjögren and Gillespie syndromes (OMIM 213300, 248800, 206700 - Online Mendelian Inheritance in Man - http://www.ncbi.nlm.nih .gov/Omim/).

An excess of children born with ataxic CP has been previously noted in the Asian population of Yorkshire compared with Caucasian children from the same region. ${ }^{9}$ We have identified a large complex consanguineous family with four affected children in two sibships suffering from congenital ataxia. All had normal pregnancies, unremarkable birth histories and no obvious underlying cause. These children all have ataxic cerebral palsy, but interestingly one of the four children also has lower limb spasticity and is classified as having ataxic diplegic cerebral palsy. Given the pedigree structure and the lack of any other obvious cause the most likely explanation is that this is an autosomal recessive trait. The presence of a child in the sibships with an ataxic diplegic phenotype is particularly interesting as this is often considered to be more like spastic diplegia than simple ataxic CP. We performed a genome-wide search to map the disease gene locus in this family.

\section{Methods \\ Study families and clinical features}

The family was ascertained from the UK Yorkshire Regional Cerebral Palsy Register, which was established in 1990 to ascertain all children at the age of 5 with a diagnosis of $\mathrm{CP}$ living within the area of the Yorkshire Regional Health Authority, a region covering approximately 3 million people. Each Asian proband affected with ataxic $C P$ was visited by one of the authors (DPM) and a single family with four affected children in two sibships was ascertained.

Sibship 1 IV-1: born at 40 weeks gestation by normal delivery after an uneventful pregnancy. Motor milestones were delayed, sitting at 10 months and walking independently at $3 \frac{1}{2}$ years of age. $\mathrm{He}$ is of normal intelligence. Examination of cerebellar function revealed dysarth ria, a stiff broad-based gait, dysdiadochokinesis, and horizontal nystagmus. The only other abnormal findings were globally decreased power in both lower limbs with increased tone, hyper-reflexia, and positive Babinski responses.

IV-2: born at 40 weeks gestation following normal delivery. Her motor milestones were also delayed as she did not sit until 10 months of age and was unable to walk independently until the age of 4 years. On examination she had dysarthria, a broad based gait an dysdiadochokinesis. There were no other abnormal neurological signs: in particular, no increase in tone or reflexes in the lower limbs. She is currently in a mainstream school and has no special problems.
Magnetic resonance imaging ( $\mathrm{MRI}$ ) was performed on both of these children, and demonstrated mild hypoplasia/atrophy of the cerebellum and spinal cord which was more marked in IV-1.

Sibship 2 IV-5 and IV-6 were born in Pakistan by normal delivery following uncomplicated pregnancies. Motor delay was noted, as in their cousins, with both children failing to sit until they were 10 months of age and being unable to walk until their 4th year. Their intelligence was within the normal range and there were no other abnormalities described. Examination revealed abnormalities of cerebellar function with dysarthria, broad based gait, dysidadochokinesis and nystagmus.

It has not been possible to perform neuroimaging on the two affected individuals in this branch of the family.

\section{Genome search}

All four affected individuals and their parents were genotyped in the initial genome search. The three unaffected individuals were then genotyped for any regions of homozygosity. The ABI PRISM ${ }^{\mathrm{TM}}$ Linkage Mapping Set Version 1.0 (Applied Biosystems) was used for the genome search. The set consists of 343 autosomal dinucleotide markers with an average spacing of $10 \mathrm{cM}$. Extra markers mapping to regions of possible homozygosity were identified from the CEPHGénéthon Web Site (http://www.cephb.fr/bio/ceph -genethon-map.html). PCR products were labelled by the incorporation of 5'-fluorescently labelled oligonucleotide primers. Analysis of the PCR products was performed using an ABI 377 Sequencer (Applied Biosystems).

PCR was performed in a total volume of $15 \mu$ l containing $40 \mathrm{ng}$ DNA, $0.2 \mathrm{~mm}$ dNTPs, $1.0-2.5 \mathrm{~mm} \mathrm{M} \mathrm{gCl}_{2}, 50 \mathrm{ng}$ of each primer, 1 unit of Taq DNA polymerase, $50 \mathrm{~mm} \mathrm{KCl,} \mathrm{10mm}$ Tris- $\mathrm{HCl}$ ( $\mathrm{pH} 9.0$ ), and $1 \%$ Triton ${ }^{\circledR} \mathrm{X}-100$. Annealing temperatures and magnesium concentrations for each primer pair were provided with the mapping set. PCR cycles were as follows: $95^{\circ}$ for $5 \mathrm{~min}$ followed by 30 cycles of $95^{\circ} \mathrm{C}$ for $20 \mathrm{~s}$, annealing temperature for $20 \mathrm{~s}, 20$ at $72^{\circ}$ with a final extension of $5 \mathrm{~min}$ at $72^{\circ} \mathrm{C}$. PCR products were analysed on a $4 \%$ polyacrylamide/ $6 \mathrm{~m}$ urea/ $1 \times$ TBE gel for $2 \mathrm{~h}$ at $3000 \mathrm{~V}$ and a fixed temperature of $51^{\circ} \mathrm{C}$. The data was captured using the $A B I$ Genescan ${ }^{T M}$ software package and genotypes generated using the Genotyper ${ }^{\mathrm{TM}}$ software package (Applied Biosystems).

\section{Statistical Analysis}

Linkage analysis was performed using both FASTLINK for two-point analysis ${ }^{10}$ and HOMOZ/MAPMAKER for multipoint analysis. ${ }^{11}$ The allele frequencies for each polymophic marker were estimated using 35 unrelated control individuals originating from the same region of Pakistan as the families involved in the study. A disease allele frequency of 1 in 100 was used. A fully penetrant autosomal recessive mode of inheritance was assumed. 


\section{Results}

Three regions of homozygosity at chromsomes $8 q$, 9cen and $19 q$ were identified in the initial genome search. Therefore additional markers in these regions were analysed in the affected individuals, their parents and unaffected sibs.

A region of homozygosity of approximately $5 \mathrm{cM}$ was demonstrated on chromosome $8 q$ between the DNA markers D8S270 and D8S514. However, two unaffected siblings were haploidentical with the affected children, which excludes this region.

A $30 \mathrm{cM}$ region of homozygosity was seen in two affected individuals from one sibship on chromosome 19q extending from D19S220 to D19S418. A further 10 markers were analysed for all the affected individuals, resulting in a $3 \mathrm{cM}$ map. No homozygous region was observed in the second sibship making this candidate region unlikely under the assumption that there is only one mutant allele in the family.

A homozygous region of approximately $23 \mathrm{cM}$ near the centromere on chromosome9 between D9S50 and D9S167 was observed in all affected individuals (Figure1). The multipoint Lod score confirmed that this region was linked to the disease phenotype (Figure2).

The maximum two point Lod generated was 2.02 with marker D9S1876 at $\theta=0$ (Table1). Multi-point analysis was performed using HOMOZ/MAPMAKER and a maximum Lod score of 3.4 was obtained between D9S50 and D9S167.

\section{Discussion}

Non-progressive congenital ataxia can be divided into ataxic $\mathrm{CP}$, ataxic diplegic $\mathrm{CP}$, dysequilibrium syndrome and Cayman Island congenital ataxia. $2,7,12$

Ingram divided ataxic CP according to the presence or absence of lower limb spasticity and termed this condition ataxic diplegic cerebral palsy. ${ }^{7}$ There is an excess of preterm infants seen with ataxic diplegic CP and it therefore resembles spastic $\mathrm{CP}$ rather than simple ataxic $\mathrm{CP}$, with respect to its aetiological factors. However, familial forms of ataxic diplegic CP are reported in the literature and the majority of these cases are inherited as an autosomal recessive trait. ${ }^{13}$
I

II

III

IV

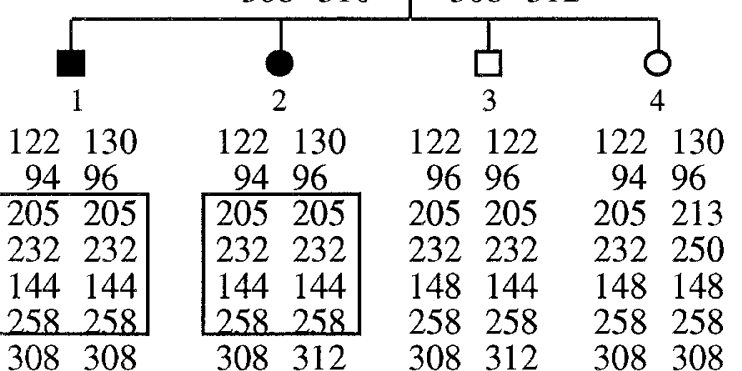

\begin{tabular}{|cl|}
\hline symbols & markers used \\
$\square$ unaffected male & D9S161 \\
0 unaffected female & D9S50 \\
D9S273 \\
a affected male & D9S166 \\
D9S1876 \\
$\bullet$ unaffected female & D9S175 \\
& D9S167 \\
\hline
\end{tabular}

Figure 1 Genotypes of the family for each marker within the linked region. The region of homozygosity for each sibship is boxed. 
Table 1 Two point Lod scores for each marker within the linked homozygous region

\begin{tabular}{llllrlll}
\hline Marker name & $\theta=0$ & $\theta=0.01$ & $\theta=0.05$ & $\theta=0.1$ & $\theta=0.2$ & $\theta=0.3$ & $\theta=0.4$ \\
\hline D9S161 & -1.99 & -0.24 & 0.27 & 0.34 & 0.23 & 0.09 & 0.01 \\
D9S50 & $-\infty$ & -1.88 & -0.66 & -0.24 & 0.02 & 0.07 & 0.05 \\
D9S273 & 1.09 & 1.06 & 0.95 & 0.80 & 0.50 & 0.23 \\
D9S166 & 1.09 & 1.06 & 0.95 & 0.80 & 0.50 & 0.23 & 0.06 \\
D961876 & 2.02 & 1.98 & 1.80 & 1.58 & 1.11 & 0.66 & 0.27 \\
D9S175 & 0.58 & 0.56 & 0.50 & 0.41 & 0.25 & 0.12 & 0.03 \\
D9S167 & $-\infty$ & -3.13 & -1.28 & -0.60 & -0.12 & 0.00 \\
\hline
\end{tabular}

Si mple ataxic CP is rarely seen in preterm infants and is due to genetic factors in approximately $50 \%$ of cases. The usual mode of inheritance is autosomal recessive, however, autosomal dominant and $\mathrm{X}$-linked forms of the disorder have also been described. ${ }^{4,7,8}$

Cayman Island congenital ataxia is a common disorder in the Cayman islands with a prevalence of approximately 1 in 150 in Grand Cayman. It manifests with marked psychomotor retardation and prominent non-progressive cerebellar dysfunction including nystagmus, intention tremor, dysarthria, and wide-based ataxic gait. ${ }^{12} \mathrm{Up}$ to one in six members of the population are carriers for the mutant allele and the condition has been mapped to chromosome 19p. ${ }^{14}$
Dysequilibrium syndrome is a form of congenital ataxia which is characterised by a variety of congenital abnormalities, including mental retardation in most cases, truncal ataxia, severely delayed motor development, muscular hypotonia, and perceptual abnormalities indicative of widespread brain dysfunction. ${ }^{2,15}$

Neuroimaging has been performed in several series of patients with non-progressive cerebellar ataxias. ${ }^{16,17}$ Generalised cerebellar hypoplasia is often seen but the degree of hypoplasia does not seem to correlate with the severity of the disorder. The imaging performed on our patients is interesting in that spinal cord hypoplasia/atrophy is seen and is most pronounced in the child with lower limb spasticity.

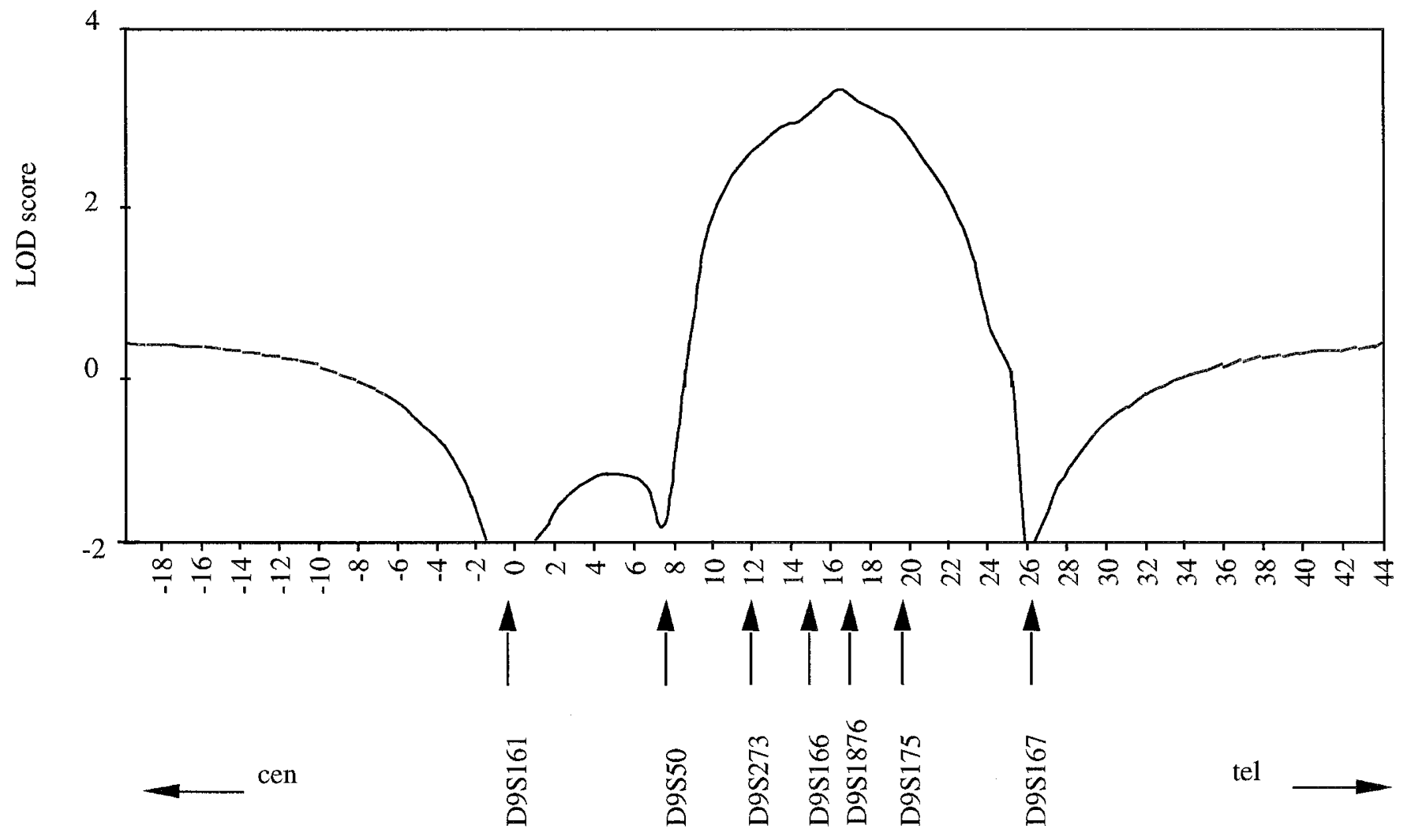

Distance in $\mathrm{cM}$

Figure 2 Multipoint Lod scores for the linked region. 
Despite increasingly sophisticated neuroimaging techniques it is not possible to classify this disorder according to their findings.

We have reported a consanguineous family containing three children affected by simple ataxic $C P$ and one child with ataxic diplegic CP. We have used homozygosity mapping to confirm that this is a single gene disorder in the family and the gene responsible for the disorder maps to chromosome 9 between markers D9S50 and D9S167, a region of approximately $23 \mathrm{cM}$. This demonstrates that these phenotypes can both result from mutations at a single locus and that the variability is either due to other gene-gene interactions or gene-environment interactions.

The maximum Lod score obtained using multipoint analysis was 3.4. A simulation of the maximum Lod score possible from this pedigree was performed using 10 fully informative markers spaced at $1 \mathrm{cM}$ intervals. The value obtained was 3.46 suggesting that the Lod score observed from the data in the study was close to the maximum predicted for this pedigree based on conservative allele frequencies.

Several further markers are now available between D9S50 and D9S167 and these will be utilised to further refine the region. In parallel, candidate genes al ready mapping into the region using the information available from the public databases will be examined for mutations using a combination of SSCP and sequencing.

Two positional candidate genes for this disorder map within the region; FRDA and PAX 5/BSAP (Genemap 98, http://www.ncbi.nlm.nih.gov/genemap98/). FRDA encodes the protein Frataxin, and mutations in this gene have been shown to cause Friedreich's ataxia. ${ }^{18}$ This is a progressive condition which classically presents before adolescence with unco-ordination of limb movements, dysarthria, nystagmus, diminished or absent tendon reflexes, and upgoing plantar responses. Other common features include scoliosis, optic atrophy with reduced visual acuity, and cardiomyopathy. The essential criteria for diagn osis are age of onset before 25 years; progressive ataxia of limbs and gait, absent knee and ankle jerks, upgoing plantar responses, and abnormal motor nerve conduction within 5 years of the diagnosis. ${ }^{19}$ An atypical form of this condition has been described with preserved reflexes (although not hyper-reflexia) and has been shown to be allelic to the typical form. ${ }^{20}$ No cases have been described with congenital onset and whilst very slowly progressive forms are described, all present later in life. ${ }^{21}$ Although the FRDA gene is a possible can didate gene because of its position and tissue expression, the phenotype in our family is significantly different from previously reported cases.

Members of the mammalian PAX (paired box) family of genes were initially identified due to sequence homology to the Drosophila segmentation genes, paired and gooseberry. ${ }^{22}$ Currently, nine members of this family of genes have been identified in humans (PAX1-PAX9). PAX genes have also been identified in mouse and zebra fish, where they are expressed in a tissue specific manner and appear to play a role in embryonic development. The PAX family is characterised by the presence of paired box domains. Pax 5 is specifically expressed in the developing mesencephalon (which goes on to form the cerebellum) and the spinal cord in mice. Whole gene knockouts of Pax 5 in mice lead to mid-brain patterning abnormalities. ${ }^{23}$

There are 167 reported ESTs in this region and of these approximately 100 have been isolated in brain tissue libraries. In addition there are 19 neurally expressed genes and all are candidates by virtue of their position in the genome (Genemap98, http://www.ncbi.nlm.nih.gov/genemap98/).

There are 32 mouse mutants described with ataxia and/or cerebellar anomalies as phenotypes in the Dysmorphic Human-Mouse Homology Database (DHMHD, http:/ /www.hgmp.mrc.ac.uk/dhmhd). The waddler mouse, meander tail mouse and the purkinjecell degeneration mouse potentially map into our region of interest (Mouse Genome Database http://www.informatics.jax.org/). Of these, the best candidate is the waddler mouse as it presents with a nonprogressive ataxia from 14 days of age and maps to within $20 \mathrm{cM}$ of the murine pax 5 . Unfortunately, this spontaneous mutant mouse model is now probably extinct. The purkinje cell degeneration mouse maps to mouse chromosome13, which has a region of synteny with human chromosome $9 q 21$. This is telomeric to our region of interest and the mouse has a different phenotype with the main feature being a progressive ataxia. The meander tail mouse maps to a region of mouse chromosome 4 which is syntenic to human chromosome 9p21. This is just telomeric of our region but again the mouse has a different phenotype, with a myopathy as well as ataxia.

Ataxic cerebral palsy has the largest genetic component of all the sub-types of cerebral palsy with up to $50 \%$ being inherited in an autosomal recessive manner. We present a family with children affected with both ataxic cerebral palsy and ataxic diplegic cerebral palsy, demonstrating that these two conditions could be caused by mutations in the same gene. In addition we have used homozygosity mapping to localise the gene to chromosome 9p12-q12. Isolation of the gene will enhance our understanding of cerebellar development and provide further genetic methods to distinguish families at high risk of having further affected children from those at much lower risk.

\section{Acknowledgements}

We thank the Wellcome Trust and the Northern and Yorkshire Regional Health Authorities for providing funding support for this research. Work in the authors' laboratories is also supported by the Medical Research Council, the Birth Defects Foundation, Yorkshire Cancer Research Campaign, Northern and Yorkshire Regional Health Authorities, and the West Riding Medical Research Trust.

\section{References}

1 Hughes I, Newton R: Genetic aspects of cerebral palsy. Dev Med Child Neurol 1992; 34: 80-86. 
2 Hagberg B, Sanner G, Steen G: Dysequilibrium syndrome in ataxic cerebral palsy. Acta Paediatr Scand 1972; 61: 1-63.

3 Volpe JJ: Neurology of the Newborn. WB Saunders: Philadelphia, 1987.

4 Gustavson KH, Hagberg B, Sanner G: Identical syndromes of cerebral palsy in the same family. Acta Paediatr Scand 1969; 58: 330-340.

5 Bundey S, Griffiths MI: Recurrence risks in families of children with symmetrical spasticity. Dev Med Child Neurol 1977; 19: 179-191.

6 Paneth N: Birth and origins of cerebral palsy. NEJM 1986; 315: 125-126.

7 Ingram TTS: Congenital ataxic syndromes in cerebral palsy. Acta Paediatr Scand 1962; 51: 209-221.

8 Young ID, Moore JR, Tripp JH: Sex linked recessive congenital ataxia. J Neurol Neurosurg Psychiatry 1987; 50: 1230-1232.

9 Sinha G, Corry P, Subesinghe D, Wild J, Levene MI: Prevalence and type of cerebral palsy in a British ethnic community: the role of consanguinity. Dev Med Child Neurol 1997; 39: 259-262.

10 Lathrop GM, Lalouel J-M: Easy calculations of Lod scores and genetic risks on small computers. Am J Hum Genet 1984; 36: 460-465.

11 Kruglyak L, Daly M, Lander E: Rapid multipoint linkage analysis of recessive traits in nuclear families, including homozygosity mapping. Am J Hum Genet.

12 Johnson WG, Murphy M, Murphy WI, Bloom AD: Recessive congenital cerebellar disorder in a genetic isolate: CPD typeVII? Neurology 1978; 28: 352-353.

13 Hagberg B, Hagberg G: The origins of cerebral palsy. In: David TJ (ed.). Recent Advances in Paediatrics. Churchill Livingstone: New York, 1993; vol 5: pp 67-83.
14 Nystuen A, Benke PJ, Merren J, Stone EM, Sheffield VC: A cerebellar ataxia locus identified by DNA pooling to search for linkage disequilibrium in an isolated population from the Cayman Islands. Hum Mol Genet 1996; 5(4): 525-531.

15 Sanner G: The dysequilibrium syndrome: a genetic study. Neuropaediatrie 1973; 4: 403-413.

16 Kornberg AJ: An extended phenotype of an early onset inherited non-progressive cerebellar. J Child N eurol 1991; 6: 20-23; 1995; 56: 519-527.

17 Sarnat HB, Alcalà H: Human cerebellar hypoplasia. Arch Neurol 1980; 37: 300-305.

18 Campuzano V, Montermini L, Molto MD et al: Friedreich's ataxia: autosomal recessive disease caused by an intronic GAA triplet repeat expansion. Science 1996; 271: 1423-1427.

19 Harding AE: The hereditary ataxias and related disorders. In: Clinical Neurology and Neurosurgery Monographs. Churchill Livingstone: New York, 1984; vol 6: 57-96.

20 Palau F, De Michele G, Vilchez JJ et al: Early-onset ataxia with cardiomyopathy and retained tendon reflexes maps to the Freidreich's ataxia locus on chromosome9p. Ann Neurol 1995; 37: 359-362.

21 Bidichandani Sl, Ashizawa T, Patel PI: Atypical Freidreich's ataxia caused by compound heterozygosity for a novel missense mutation and the GAA triplet-repeat expansion. Am J Hum Genet 1997; 60: 1251-1256.

22 Burri M, Tromvoukis Y, Bopp D, Frigerio G, Noll M: Conservation of the paired domain in metazoans and its structure in three isolated human genes. EMBO J 1989; 8(4): 1183-1190.

23 Urbanek P, Wang ZQ, Fetka I, Wagner EF, Busslinger M: Complete block of early $B$ cell differentiation and altered patterning of the posterior midbrain in mice lacking Pax5/BSAP. Cell 1994; 79(5): 901-912. 